\title{
APPROACHES TO MODELING OF COGNITIVE EVOLUTION
}

\author{
Vladimir G. Red'ko \\ Scientific Research Institute for System Analysis, \\ Russian Academy of Science, \\ 44/2, Vavilova Str., Moscow, 119333, Russia, \\ vgredko@gmail.com, http://www.niisi.ru/iont/staff/rvg/index_eng.php
}

\begin{abstract}
Approaches to modeling of cognitive evolution that is evolution of animal cognitive abilities are proposed and discussed. Backgrounds of models of cognitive evolution, that are developed in an area of researches "Adaptive behavior", in which modeled "organisms" adapting to variable environment are studied, are outlined. Initial steps of modeling of cognitive evolution are characterized. The sketch program for future investigations of cognitive evolution is proposed.
\end{abstract}

Keywords: cognitive evolution, modeling, adaptive behavior, animal cognitive abilities.

\section{INTRODUCTION}

Approaches to modeling of cognitive evolution are proposed and discussed in the current paper. Modeling of cognitive evolution is a study of evolution of animal cognitive properties by means of mathematical and computer models. This modeling is related to epistemological problems, to foundations of mathematics, to the problem of human intelligence origin. Some initial steps towards development of models of cognitive evolution have been made in the area of research "Adaptive Behavior". Investigations of adaptive behavior are described shortly below.

We begin with discussion of the epistemological problem: why human intelligence is applicable to cognition of nature? This problem and approaches to analyze it by means of investigation of cognitive evolution are described in Section 2. Then we describe the area of research "Adaptive Behavior" (Section 3) and typical models of adaptive behavior (Section 4). Models of adaptive behavior that are directly related to cognitive evolution are outlined in Section 5. The sketch program for future modeling of cognitive evolution is proposed in Section 6 . Section 7 underlines the importance of modeling of cognitive evolution. Section 8 concludes the paper.

\section{EPISTEMOLOGICAL PROBLEM}

There is the profound epistemological problem: why the human thinking is applicable to cognition of the nature? To characterize the problem, let us consider physics. The power of physics is due to effective use of mathematics. However, why mathematical deductions are applicable to studies of real physical phenomena? Indeed, a mathematician makes logical inferences, proves theorems, basing on his mind, independently from the physical world. Why his results are applicable to the real nature?

Similar questions were interesting for scientists for a long time. In the 1780 s, Immanuel Kant investigated human thinking, human cognition. As a result, the well-known "Critique of pure reason" [1] and its popular narration "Prolegomena to any future metaphysics" [2] appeared. Kant investigated cognitive abilities in a certain approximation, namely, in the approximation of single adult human person. He did not analyze the origin of human cognitive abilities, he presumed that these abilities exist and analyzed, how these abilities operate in scientific cognition of the nature. According to Kant, there is a system of categories, concepts, logic rules, and inference methods, which are used in cognition of nature. This system of "pure reason" is of a priory character; it exists in our minds before any experience. Analyzing scientific cognition of nature, Kant concluded that as pure reason is of a priory character, our reason prescribes its laws to nature [2]:

"...it seems at first strange, but is not the less certain, to say: the understanding does not derive its laws (a priori) from, but prescribes them to, nature."

Probably, it was reasonable in Kant's times to 
use the approximation of single adult person: it is difficult to analyze all sides of the problem at once. Besides, Charles Darwin's theory of species origin was not created yet. After appearance of Darwinian theory, the concept of a priory "pure reason" had to be revised. And the revision did occur. It was clearly expressed by Konrad Lorenz in the paper "Kant's doctrine of the a priori in the light of contemporary biology" [3].

According to Lorenz, Kantian a priory categories and other forms of "pure reason" were emerged during natural selection. Components of "pure reason" arose during evolution, as a result of numerous interactions with the external world. In an evolutionary context, "pure reason" is not of a priory character, it has obvious evolutionary empirical roots.

Actually, Kant and Lorenz demonstrated that without analysis of evolutionary origin of human cognitive abilities, there is no answer to the question of applicability of the human thinking to cognition of the nature.

In order to analyze evolutionary roots of human mind, it is natural to investigate, why and how high level cognitive abilities did emerge during evolutionary processes. In other words, we can follow evolutionary roots of animal and human cognitive abilities and represent a general picture of evolutionary emergence of the human thinking.

Can we really proceed in this way? Can we find evolutionary roots of the human mind, analyzing animal cognition properties? Yes, we can. In order to demonstrate such possibility, we present the following analogy.

Let us consider the elementary logic rule that is used by a mathematician in deductive inferences, namely, modus ponens: "if $A$ is present and $B$ is a consequence of $A$, then $B$ is present", or

$$
\frac{A, A \rightarrow B}{B}
$$

Now let us go from the mathematician to a dog that is subjected to the experiment of classical conditioning. A neutral conditioned stimulus (CS) precedes a biologically significant unconditioned stimulus (US). After a number of presentations of the pair (CS, US), the causal relation CS $\rightarrow$ US is stored in the dog's memory. Using this relation at a new presentation of the CS, the dog is able to do the elementary "inference":

$$
\frac{\mathrm{CS}, \mathrm{CS} \rightarrow \mathrm{US}}{\mathrm{US}} .
$$

Thus, after the presentation of the CS, the dog expects the US.

Of course, the application of the rule modus ponens (purely deductive) by the mathematician and the inductive "inference" of the dog are obviously different. However, can we think about evolutionary roots of logical rules that are used in mathematics? Yes, we certainly can. The logical conclusion of the mathematician and the inductive "inference" of the dog are similar. Thus, this analogy demonstrates that we can analyze evolutionary roots of the human thinking.

It should be noted that the final results of cognitive evolution, the logical rules that are used in mathematical deductions are known and formalized. For example, in the 1930s, Gerhard Gentzen developed methods of natural deduction and formalized sets of logical rules that are used at theorem proofs [4]. These sets of logical rules are based on elementary inference rules such as modus ponens.

How can we concretely investigate origin of logic forms of thinking? Are any backgrounds for modeling of cognitive evolution? Fortunately, there is an area of research "Adaptive Behavior" that includes some initial steps of modeling of cognitive evolution. This field of investigations is characterized in the next section.

\section{AREA OF INVESTIGATIONS "ADAPTIVE BEHAVIOR"}

In the early 1990 s, the area of investigations "Adaptive Behavior" was initiated [5]. The basic approach of this research direction is designing and investigation of artificial (in the form of a computer program or a robot) "organisms" that are capable to adapt to a variable environment. These organisms are often called "animats" or agents, autonomous agents. The term "animat" originates from two words: animal + robot $=$ animat.

The ultimate goal of this field of research is "...to embed human intelligence within an evolutionary perspective and to seek how the highest cognitive abilities of man can be related to the simplest adaptive behaviors of animals" [6]. This goal is similar to the goals of modeling of cognitive evolution.

Investigations of adaptive behavior are based on serious computational intelligence methods:

- $\quad$ Neural networks,

- $\quad$ Genetic algorithm [7] and other methods of evolutionary computations,

- $\quad$ Classifier Systems [8],

- $\quad$ Reinforcement Learning [9].

Applications of this direction of research are artificial intelligence, robotics, models of adaptive behavior in social and economic systems. 
Typical examples of models of adaptive behavior are outlined in the next section.

\section{EXAMPLES OF MODELS OF ADAPTIVE BEHAVIOR}

\subsection{MODELS OF BRAIN AND BEHAVIOR AT THE NEUROSCIENCE INSTITUTE}

At the Neuroscience Institute (http://www.nsi.edu) that is leaded by Gerald Edelman, a number of models of a brain (Darwin I, Darwin II, ...) are investigated. These models are used to construct control systems of artificial organisms NOMAD (Neurally Organized Mobile Adaptive Device) or Brain-Based Device.

Principles of NOMAD modeling are as follows:

1) The device NOMAD is located in the real physical environment.

2) The device should solve certain behavioral problem.

3) The nervous system of the device should reflect architecture of a brain and dynamics of processes in a brain.

4) The behavior of the device and processes in modeled nervous system should be compared with experimental biological data.

Behavior of a mouse in a Morris water maze has been modeled in [10]. Investigation of a mouse or a rat in the Morris water maze is one of modern canonical biological experiments. This experiment consists in the following. There is a pool with an opaque liquid (for example, it can be water that is colored slightly by milk). There are different drawings on pool boards; the mouse sees and can use these drawings for orientation. A hidden platform is in a certain place of the pool. The mouse can find the platform and escape from sinking. The experimenter throws the mouse into the pool, the mouse floats some time and then it finds the platform and escapes from sinking or starts to sink (then it is rescued by the experimenter). After a number of experiments the mouse begins to use drawings on pool boards for orientation and to find the platform rather quickly.

Behavior of a mouse in a Morris water maze has been modeled by means of NOMAD as follows [10]. NOMAD was the wheeled mobile device; its control system was a neural network that consisted from 90000 neurons. This neural network had 50 different various neural areas; in particular, certain areas of a hippocampus were distinguished. The neural network had $1.4 \cdot 10^{6}$ synaptic contacts between neurons. The processes that occurred in different neural network areas were investigated in details. The NOMAD sensory system consisted from a camera for vision, an odometry for self-movement cues, a system of infrared transceivers for obstacle avoidance, and the special infrared detector of the hidden platform; this detector was able to detect the platform only for such time moments, when NOMAD was directly over the platform.

NOMAD was located in a room that had the hidden platform; different multi-colored strips (drawings) were on room walls. In the beginning of each of computer experiments, NOMAD was located in different sites of the room; the task of NOMAD was to find the hidden platform. Learning of the neural network of NOMAD was accomplished by means the modified Hebb rule. The modification is based on reinforcement learning [9]. Reinforcement learning used rewards (received by NOMAD at approaching to the hidden platform) and punishments (received at approaching to room walls).

The simulation demonstrated that 1) NOMAD was trained to find the platform quickly (during 1020 modeled experiments); 2) place neurons were formed in the modeled hippocampus; these neurons were active, only when NOMAD was in certain places of the room; 3) certain causal connections between some neural areas were formed in the modeled hippocampus.

Thus, the described simulation is interesting biologically inspired computer research of the selflearning adaptive device.

\subsection{BIONIC MODEL OF SEARCHING ADAPTIVE BEHAVIOR}

Interesting direction of researches within the framework of "Adaptive Behavior" is a study of searching animal behavior. Searching behavior of caddis fly larvae Chaetopteryx villosa was modeled in [11]. Larvae inhabit creek bottoms and carry on themselves a case ("a small house") that is a tube from sand and other particles which they collect at the creek bottom. Larvae build their cases from hard particles; they can use small or large sand particles. Using large particles, the larva can build cases more quickly and effectively than with small particles, so its preference is evident. Large particles are distributed randomly, but typically occur in groups of several particles. However, search of large particles at the creek bottom demands time and energy expenses, not known to the larva in advance.

The larva uses two tactics: 1) testing particles in its vicinity and building the case from selected particles, 2) searching for a new place with a collection of appropriate particles. Investigations of larva behavior reveal inertia in switching between tactics.

The computer model of searching behavior of larvae is designed in [11]. The model uses a concept 
of a motivation, namely the motivation to an attachment of particles to a larva case. Dynamics of the motivation $M(t)$ is described by the equation:

$$
M(t)=k_{1} M(t-1)+\xi(t)+I(t),
$$

where $t$ is discrete time, $t=1,2, \ldots, k_{1}$ is the parameter characterizing a slow relaxation of the motivation $\left(0<k_{1}<1,1-k_{1}<<1\right), \xi(t)$ is the random variation of the motivation, the variable $I(t)$ characterizes the directed change of the motivation at testing of particles by the larva:

$$
I(t)=k_{2} \frac{S_{\text {curr }}-S_{\text {last }}}{S_{\text {last }}},
$$

where $k_{2}$ is the positive parameter, $S_{\text {curr }}$ is the area of the currently tested particle, $S_{\text {last }}$ is the area of the last particle that has been tested before.

If the motivation $M(t)$ is sufficiently large, then the testing and the attachment of particles to the larva case occur; this behavior corresponds to the first tactic of behavior. If the value $M(t)$ is small, then the larva moves and searches for a new place with suitable sizes of particles; this corresponds to the second tactic of behavior.

The developed model [11] agrees satisfactorily with biological experimental data. Both in biological experiments and in the model, large particles are preferably attached to the case; there is a large distribution in number of attached particles; the total number of attached particles is not large in both cases.

It should be noted that according to (3), (4), dynamics of the motivation $M(t)$ is simple and effective. This dynamics takes into account the inertia of the motivation change, random variations and the directed change of the motivation $M(t)$. So, the developed approach can be used at modeling of a regulation of switching between behavioral tactics of animals that have several needs and corresponding motivations. This approach can be also used for development of artificial animat control systems, for example, for development of control systems of mobile robots.

\subsection{MODEL OF EVOLUTION OF POPULATION OF AUTONOMOUS ADAPTIVE AGENTS}

The computer model of agents which are similar to the biological organisms adapting to change of temperature $T$ in the external environment is designed and analyzed. The control system of an agent is based on neural network adaptive critic designs [12]. The control system ensures the forecasting of $T$ changes and the agent movement in accordance with temperature changes. Agent behavior is adjusted by means of reinforcement learning and evolutionary optimization. The interaction between learning and evolution is analyzed. The Baldwin effect is demonstrated in the current model: certain acquired features (obtained by means of learning) of agents can be genetically assimilated during several generations of Darwinian evolution.

The Baldwin effect [13], [14] that is the genetic assimilation of acquired features during a number of generations of Darwinian evolution is well known. The operation of this effect includes two stages. At the first stage, evolving organisms obtain (owing to appropriate mutations) a property to learn some useful features. Fitness of such organisms increases; hence, they are distributed in the population. But learning has some disadvantages: it demands energy and time. Therefore the second stage (the genetic assimilation) is possible: useful features can "be reinvented" by evolutionary processes and these features can be directly coded in genomes of organisms.

\subsubsection{DESCRIPTION OF THE MODEL}

The model is based on the following analogy. Modeled "lizards" that adapt to temperature changes are considered. The adaptation essence consists in the following. There are two places, which lizards can choose: 1) a place on a stone, 2) a place in a burrow. The natural behavior is as follows. At large temperature the lizard heats on the stone, at low temperature it gets into the burrow and keeps its body warm.

The agent-lizard uses its control system to choose a place. The agent control system is optimized by means of reinforcement learning and Darwinian evolution.

The temperature of the external environment $T_{\text {ext }}$ (the temperature on the stone) is determined by time series $T_{e x t}(t), t=1,2, \ldots$ The current situation $\mathbf{S}(t)$ is determined by two values $T_{e x t}(t)$ and $P(t), \mathbf{S}(t)=$ $\left\{T_{\text {ext }}(t), P(t)\right\}$, where $P(t)$ is the parameter of the position of the lizard: $P(t)=0$ if the lizard is in the burrow, and $P(t)=1$ if the lizard is on the stone. The action of the lizard is the choice of its position $P(t+1)$ in the next time moment.

It is supposed that there is some optimum temperature of lizard body $T_{0}$ and when the lizard is in the burrow its temperature is close to $T_{0}$; though the environment temperature influences slightly on the temperature in the burrow. So, the temperature in the burrow $T_{\text {int }}(t)$ is

$$
T_{\text {int }}(t)=T_{0}+k_{3}\left[T_{\text {ext }}(t)-T_{0}\right],
$$


where $k_{3}$ is the small positive parameter, $0<k_{3}<<1$.

The reinforcement $r(t)$, which is received by the lizard at the time moment $t$, is proportional to the difference $T(t)-T_{0}$, where $T(t)$ is the current temperature in that place where the lizard is in the moment $t$ :

$$
r(t)=k_{4}\left[T(t)-T_{0}\right],
$$

where $k_{4}>0$. For simplicity we suppose that the lizard predicts $T_{\text {ext }}(t)$, and $T_{i n t}(t)$ can be estimated by it according to the Eq. (5).

Control system of the agent-lizard. The control system of the agent-lizard is intended for maximization of the utility function $U(t)[9]$ :

$$
U(t)=\sum_{j=0}^{\infty} \gamma^{j} r(t+j), \quad t=1,2, \ldots
$$

where $r(t)$ is the current reinforcement determined by the Eq. (6), $\gamma$ is the discount factor $(0<\gamma<1$, $1-\gamma<<1)$.

The control system of the agent consists of two neural networks (NNs): the model and the critic. The model NN predicts dynamics of the environment temperature $T_{\text {ext }}(t)$. The critic $\mathrm{NN}$ estimates the utility function $U$ for the current situation $\mathbf{S}(t)$, for predicted situations for two possible positions of the agent in the next time step, and for the next situation $\mathbf{S}(t+1)$.

Operation and learning of the agent control system. Inputs of the model $\mathrm{NN}$ are $m$ previous values of temperature $T_{\text {ext }}(t-m+1), \ldots, T_{\text {ext }}(t)$, this NN predicts the environment temperature in the next time moment $T_{e x t}^{p r}(t+1)$. The model is the twolayer NN that operates according to formulas:

$$
\begin{aligned}
& \mathbf{x}^{\mathbf{M}}=\left\{T_{\text {ext }}(t-m+1), \ldots, T_{\text {ext }}(t)\right\}, \\
& y_{j}^{M}=\tanh \left(\sum_{i} w_{i j}^{M} x_{i}^{M}\right), \\
& T_{\text {ext }}^{p r}(t+1)=\sum_{j} v_{j}^{M} y_{j}^{M},
\end{aligned}
$$

where $\mathbf{x}^{\mathbf{M}}$ is the input vector, $\mathbf{y}^{\mathbf{M}}$ is the vector of outputs of neurons of the hidden layer, $w_{i j}^{M}$ and $v_{j}^{M}$ are synaptic weights of the model NN.

The critic NN is intended for the estimation of quality of a situation $\mathrm{V}(\mathbf{S}(t))$ that is the estimation of the utility function $U(t)$ for the agent in the situation $\mathbf{S}(t)$. The critic is the two-layer $\mathrm{NN}$ that operates according to formulas:

$$
\begin{aligned}
& \mathbf{x}^{\mathbf{C}}=\mathbf{S}(t)=\left\{T_{e x t}(t), P(t)\right\}, \\
& y_{j}^{C}=\tanh \left(\sum_{i} w_{i j}^{C} x_{i}^{C}\right), \\
& V(t)=V(\mathbf{S}(t))=\sum_{j} v_{j}^{C} y_{j}^{C},
\end{aligned}
$$

where $\mathbf{x}^{\mathbf{C}}$ is the input vector, $\mathbf{y}^{\mathbf{C}}$ is the vector of outputs of neurons of the hidden layer, $w_{i j}^{C}$ and $v_{j}^{C}$ are synaptic weights of the critic NN.

Following operations are carried out in the agent control system each time moment $t$ :

1) The model $\mathrm{NN}$ predicts the external temperature in the next time moment $T_{e x t}^{p r}(t+1)$.

2) The critic NN estimates the value $V$ for the current situation $V(t)=V(\mathbf{S}(t))$ and for predicted situations for both possible actions $V_{P}^{p r}(t+1)=$ $V\left(\mathbf{S}_{\mathbf{P}}^{\mathbf{p r}}(t+1)\right), \quad$ where $\quad \mathbf{S}_{\mathbf{P}}^{\mathbf{p r}}(t+1) \quad=$ $\left\{T_{\text {ext }}^{p r}(t+1), P(t+1)\right\}, P(t+1)=0$ or $P(t+1)=1$.

3) The $\varepsilon$-greedy method is applied [9]: the action corresponding to the maximum value $V_{P}^{p r}(t+1)$ is chosen with probability $1-\varepsilon$, the alternative action is chosen otherwise $(0<\varepsilon<<1)$. The action choice is the selection of the value $P(t+1)$.

4) The chosen action $P(t+1)$ is carried out. The transition to the next time moment $t+1$ occurs. The reinforcement $r(t+1)$ in accordance with the Eq. (6) is obtained by the agent. The real value $T_{e x t}(t+1)$ is observed and compared with the prediction $T_{e x t}^{p r}(t+1)$. The synaptic weights of the model NN are adjusted to minimize the error of this prediction by means of the usual back-propagation method [15]. The learning rate of the model NN is equal to $\alpha_{M}$

5) The quality of the next situation is estimated by the critic $\mathrm{NN}: V(t+1)=V(\mathbf{S}(t+1)) ; \mathbf{S}(t+1)=$ $\left\{T_{\text {ext }}(t+1), P(t+1)\right\}$. The time difference error $\delta(t)$ is calculated [9]:

$$
\delta(t)=r(t+1)+\gamma V(t+1)-V(t) .
$$

6) The synaptic weights of the critic $\mathrm{NN}$ are adjusted to minimize the time difference error $\delta(t)$; this adjustment is carried out by means of the gradient method, similar to the back-propagation method. The learning rate of the critic $\mathrm{NN}$ is equal to $\alpha_{C}$.

The evolution scheme. In addition to agent learning, the evolutionary optimization of control 
systems of agents takes place. The evolving population consists of $n$ agents. Each agent has its resource $R(t)$ that changes according to reinforcements: $R(t+1)=R(t)+r(t)$, where $r(t)$ is determined by the Eq. (6). Evolution passes through a number of generations, $n_{g}=1,2, \ldots$ Duration of each generation $n_{g}$ is $T_{g}$ time steps ( $T_{g}$ is the lifetime of the agent). At the beginning of each generation, initial resource of any agent $R(t)$ is zero. At the end of each generation, the agent having maximum resource $R_{\max }\left(n_{g}\right)$ (the best agent of the generation $n_{g}$ ) is determined. This best agent gives birth to $n$ descendants that constitute the next generation.

Each agent has two sets of synaptic weights of both NNs: $\mathbf{G}$ and $\mathbf{W}$. The set $\mathbf{G}$ are initial NN synaptic weights that are received by the agent at the moment of its birth from the agent-parent. This set $\mathbf{G}$ is the agent genome that does not vary during its life. The set $\mathbf{W}$ are temporary NN synaptic weights that are adjusted during the agent life by means of learning. At the moment of the agent birth $\mathbf{W}=\mathbf{G}$. Descendants of the agent inherit its genome $\mathbf{G}$ (with small mutations). As the genome $\mathbf{G}$ is inherited, the evolution process has Darwinian character.

\subsubsection{RESULTS OF SIMULATIONS}

The main parameters of computer simulations are the following: the discount factor $\gamma=0.9$; the number of inputs of the model $\mathrm{NN} m=10$; the number of neurons in the hidden layers of the model and critic NNs $N_{h M}=N_{h C}=10$; the learning rate of the model and critic NNs $\alpha_{C}=\alpha_{C}=0.01$; the parameter of the $\varepsilon$-greedy method $\varepsilon=0.05$; the intensity of mutations $P_{m u t}=0.1$; the duration of a generation $T_{g}=1000$, the population size $n=10$.

The time dependence of the external temperature is the sinusoid:

$$
T_{\text {ext }}(t)=0.5 \sin (2 \pi t / 20)+T_{0}, T_{0}=1.5 \text {. }
$$

In order to compare learning and evolutionary optimization the following cases were analyzed:

Case L (pure learning); in this case the single self-learning agent was considered;

Case E (pure evolution), i.e. the evolving population of agents without learning;

Case LE (learning + evolution), i.e. the full model described above.

The values of resource obtained by agents during 1000 time steps for these three cases were compared. For cases $\mathrm{E}$ and LE, the generation duration was $T_{g}$ $=1000$, and the maximum value of agent resource in the population $R_{\max }\left(n_{g}\right)$ at the end of each generation was registered. In the case L (pure learning), the single agent was analyzed. The resource of this agent was set to be zero every $T_{g}=1000$ time steps: $R\left(T_{g}\left(n_{g}-1\right)+1\right)=0$. In this case the index $n_{g}$ was increased by 1 after every $T_{g}$ time steps, and it was set $R_{\max }\left(n_{g}\right)=R\left(T_{g} n_{g}\right)$.

The plots $R_{\max }\left(n_{g}\right)$ are shown in Fig. 1 that demonstrates that learning together with evolution (the case LE), ensures more effective growth $R_{\max }$ as compared with learning or evolution separately (cases L and E). The curves are averaged over 1000 simulations.

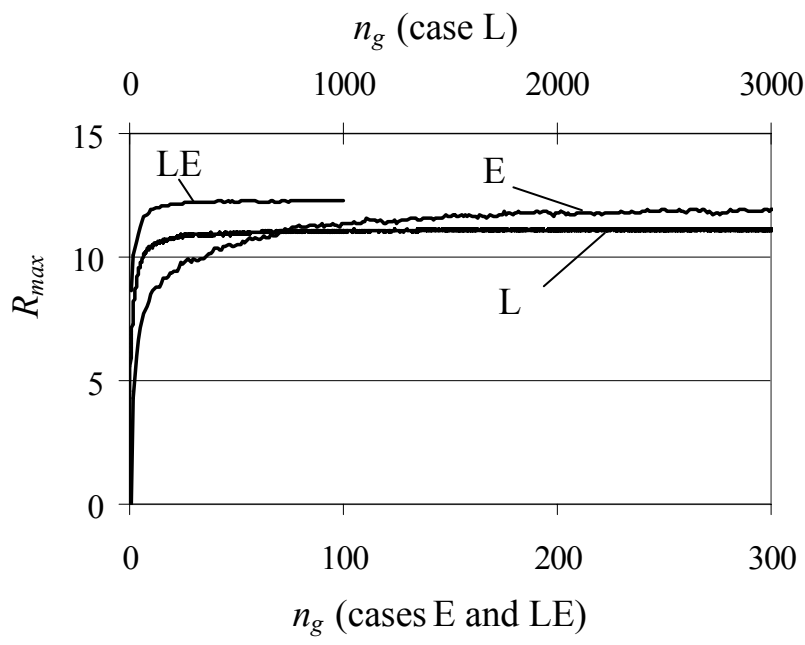

Fig. 1 - The plots $R_{\max }\left(n_{g}\right)$

The obvious influence of leaning on evolutionary processes is often observed in some simulations. In these simulations, the essential growth of resource of the best agent of the population begins with certain time delay (200-500 time steps). This means that the agent initially learns to get satisfactory behavioral policy, and only after several generations the agent resource growth begins from the start of a generation. This phenomenon can be interpreted as the Baldwin effect: the initially acquired (via learning) property to obtain resource become the inherit one during several generations. The example of this phenomenon is shown in Fig. 2. This figure demonstrates resource dynamics $R(t)$ for the best agent of the population during five generations.

Fig. 2 shows that during early generations (generations 1 and 2), any significant increase of agent resource begins only after a lag of 200 to 500 time steps. The best agent does optimize its policy by learning. Subsequently, the best agents find an advantageous policy faster and faster. By the fifth generation, a newborn agent "knows" a decent policy as it is encoded in its genome $\mathbf{G}$, and the learning does not improve the policy significantly. Thus, Fig. 2 demonstrates that the initially learned policy becomes inherited (the Baldwin effect [13], [14]).

It should be noted that the processes of agent learning and evolutionary optimization of agent control systems include random components. So, a particular dependence $R(t)$ can be different from the dependence shown in Fig. 2. However, some delays of increase of agent resource are observed in main 
part of computer experiments. The simulation represented in Fig. 2 shows profound example of such delay.

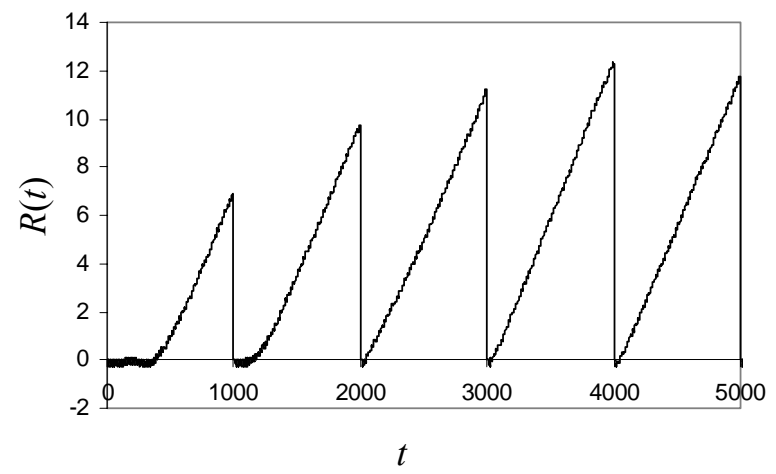

Fig. 2 - Time dependence of the resource $R(t)$ of the best agent of the population during five generations. The case LE

Thus, according to our simulations for Darwinian evolution, the good agent strategy of behavior that is initially obtained by means of learning can become inherited. Moreover, this genetic assimilation of initially acquired features can occur quickly: during only 3-5 generations. This phenomenon is important from biological point of view, because it demonstrates that some Lamarckian properties can be observed during Darwinian evolution. It should be noted that our model is rather formal. In order to understand this phenomenon in full extent, more biologically well-grounded models should be developed and investigated.

\section{MODELS OF ANIMAL COGNITIVE ABILITIES}

Certain models of cognitive abilities of animals are already investigated in the framework of "Adaptive behavior". Let us note some of such researches.

Models of conditioned reflexes were investigated in early works [16], [17].

Researches of an anticipatory behavior, at which animals predict future situations and actively use these predictions for the organization of the behavior, are conducted currently [18].

Interesting works are devoted to the formalization of rules of decision making. For example, Mark Witkowski proposed a theory of decision making rules that correspond to different levels of biological evolution [19]. Rules take into account an associative memory, conditioned reflexes, predictions of action results. Schemes of learning and decision making that are based on these rules are developed; certain computer simulations confirm efficiency of proposed rules.

Some works analyze evolutionary development of neural structures of animal brains. For example, the work [20] analyzes an evolution of neural structures that have the important role at the action selection ensuring adaptive behavior.

Thus, certain models of cognitive features of animal adaptive behavior are designed and investigated already. However, these investigations are preliminary in many aspects. The next section proposes key steps for future modeling of cognitive evolution.

\section{SKETCH PROGRAM FOR FURTHER RESEARCH OF COGNITIVE EVOLUTION}

The sketch program for further researches of cognitive evolution can include the following steps.

A) Modeling of adaptive behavior of animats that have several natural needs: food, reproduction, safety.

Such modeling can be simulations of a natural and well-developed behavior of simple modeled organisms. Modeling in this direction is already initiated (see below).

B) Investigation of the transition from the physical level of information processing in nervous system of animals to the level of generalized "notions".

Such transition can be considered as emergence of the property of "notion" in animal minds. The generalized "notions" are mental analogues of our words, which are not said by animals, but really used by them. For example, the dog obviously has internal notions "friend", "enemy", "food". Usage of notions leads to essential reduction both the needed memory and the time of information processing, therefore it should be evolutionary advantageous.

C) Investigations of processes of generating of causal relations in animal memory.

Storing relationships between the cause and the effect and the adequate use of these relationships is one of key properties of active cognition of regularities of the external world by animals. For example, such relationships are generated at the conditioned reflex: the animal remembers the temporal relation between the conditioned stimulus (CS) and the unconditioned stimulus (US). This allows it to predict events in the external world and adequately use these predictions.

Natural next step is the transition from memorizing separate causal relations to systems of logic conclusions.

D) Investigations of "logic conclusions" in animal minds.

Actually, at classical conditioning, animals do a "logic conclusion": $\{\mathrm{CS}, \mathrm{CS} \rightarrow \mathrm{US}\}=>$ US or "If the conditioned stimulus takes place, and the conditioned stimulus result in the unconditioned one, then the occurrence of the unconditioned stimulus is 
expected". We can even state that such conclusions are similar to logical conclusions at mathematical deductions (see Section 2 above). It is important to understand, how systems of these conclusions operate, to what extent this "animal logic" is similar to our human logic.

The listed items outline steps of possible investigations from simplest forms of adaptive behavior to logical rules that are used at mathematical deductions.

Basing on these steps, we began corresponding modeling [21]. The formal model of the simple agents which have needs of food, reproduction, and safety (Step A) has been designed and analyzed. The model demonstrated a natural behavior of agents. Also the important role of reproduction during evolutionary optimization of agent control systems has been revealed. It is interesting to note that similar works of other researchers appeared recently. For example, the adaptive behavior of agents that have several needs and corresponding motivations was analyzed in [22].

Another model that is described in [21] demonstrates the formation of several generalized heuristics by the self-learning agent searching food in the two-dimensional cellular environment. These heuristics result in generating chains of actions by the agent. Additionally, the formation of internal generalized "notions" by the agent (Step B) was observed in this model [23]. These notions are used by the agent in its heuristics. It should be noted that simple models described in [21], [23] can be considered as the certain initial stage of more powerful investigations of cognitive evolution.

Comparing steps of the sketch program with noted works [16-23], it is possible to conclude that there are some small elements corresponding to each step of the program already. In other words, we can see some small fragments of a picture of cognitive evolution now, but we do not see the whole picture yet. Nevertheless, investigations of cognitive evolution are interesting and important.

\section{IMPORTANCE OF MODELING OF COGNITIVE EVOLUTION}

Let us underline interdisciplinary relations and the importance of investigations of cognitive evolution.

- These investigations are related to foundations of science, to foundations of mathematics, to the serious problem: why formal logic conclusions, mathematical deductions are applicable to the real world.

- These researches are interesting from philosophical, epistemological point of view; they are aimed at understanding the applicability of the human thinking for investigations of the real physical world.

- The research area "Adaptive behavior" includes certain backgrounds for creating and developing of models of cognitive evolution.

- The investigations of cognitive evolution are interesting from the point of view of cognitive sciences, as they are connected with important cognitive processes, processes of scientific cognition.

\section{CONCLUSION}

Thus, approaches to modeling of cognitive evolution have been proposed and discussed. This modeling is related to foundations of science and to foundations of mathematics. Initial steps towards modeling of cognitive evolution have been already done in the research area "Adaptive Behavior". The sketch program for further modeling of cognitive evolution is proposed. The program includes research steps that are aimed for investigations from simple animal cognitive abilities to mathematical deductions.

\section{ACKNOWLEDGEMENTS}

This work is partially supported by the Russian Foundation for Basic Research, Grant No 10-0100129.

\section{REFERENCES}

[1] I. Kant. Kritik der reinen Vernunft, Hartknoch, Riga, 1781. See also: I. Kant, Critique of Pure Reason, trans. Werner Pluhar. Indianapolis: Hackett, 1996.

[2] I. Kant. Prolegomena zu einer jeder künftigen Metaphysik, die als Wissenschaft wird auftreten können. Hartknoch, Riga, 1783. See also: I. Kant, Prolegomena to Any Future Metaphysics, trans. Gary Hatfield. New York: Cambridge University Press, 1997.

[3] K. Lorenz. Kant's doctrine of the a priori in the light of contemporary biology. (1941), in Learning, Development and Culture: Essays in Evolutionary Epistemology, H. Plotkin, Ed. New York: Wiley, 1982, pp. 121-143.

[4] M.E. Szabo. Collected Papers of Gerhard Gentzen. Amsterdam: North-Holland Pub. Co., 1969.

[5] From Animals to Animats. Proceedings of the First International Conference on Simulation of Adaptive Behavior. J.-A. Meyer and S.W. Wilson, Eds. Cambridge: MIT Press, 1991.

[6] J.Y. Donnart, J.-A. Meyer. Learning reactive and planning rules in a motivationally autonomous animat. IEEE Transactions on 
Systems, Man, and Cybernetics - Part B: Cybernetics 26 (3) (1996). pp. 381-395.

[7] J.H. Holland. Adaptation in Natural and Artificial Systems. Ann Arbor, MI: The University of Michigan Press, 1975 (1st edn.). Boston, MA: MIT Press, 1992 (2nd edn.).

[8] J.H. Holland, K.J. Holyoak, R.E. Nisbett, P. Thagard. Induction. Processes of Inference, Learning, and Discovery. Cambridge: MIT Press, 1986.

[9] R. Sutton, A. Barto. Reinforcement Learning: An Introduction. Cambridge: MIT Press, 1998.

[10] J.L. Krichmar, A.K. Seth, D.A. Nitz, J.G. Fleischer, G.M. Edelman. Spatial navigation and causal analysis in a brain-based device modeling cortical-hippocampal interactions. Neuroinformatics 3 (3) (2005). pp. 197-221.

[11] V.A. Nepomnyashchikh, E.E. Popov, V.G. Red'ko. A bionic model of adaptive searching behavior. Journal of Computer and Systems Sciences International 47 (1) (2008). pp. 78-85.

[12] D.V. Prokhorov, D.C. Wunsch. Adaptive critic designs, IEEE Transactions on Neural Networks 8 (5) (1997). pp. 997-1007.

[13] J.M. Baldwin. A new factor in evolution. American Naturalist 30 (354) (1896). pp. 441451.

[14] Evolution, Learning, and Instinct: 100 Years of the Baldwin Effect. P. Turney, D. Whitley, R. Anderson, Eds. Special Issue of Evolutionary Computation on the Baldwin Effect 4 (3) (1996).

[15] D.E. Rumelhart, G.E. Hinton, R.G. Williams. Learning representation by back-propagating error, Nature 323 (6088) (1986). pp. 533-536.

[16] S. Grossberg. Classical and instrumental learning by neural networks. Progress in Theoretical Biology, R. Rosen, F. Snell, Eds., New York: Academic Press, vol. 3, 1974, pp. 51-141.

[17] A.G. Barto, R.S. Sutton. Simulation of anticipatory responses in classical conditioning by neuron-like adaptive element. Behavioral Brain Research 4 (3) (1982). pp. 221-235.
[18] Anticipatory Behavior in Adaptive Learning Systems: From Brains to Individual and Social Behavior. LNAI 4520, M.V. Butz, O. Sigaud, G. Pezzulo, G. Baldassarre, Eds. Berlin, Heidelberg: Springer Verlag, 2007.

[19] M. Witkowski. An action-selection calculus. Adaptive Behavior 15 (1) (2007). pp. 73-97.

[20] T.J. Prescott. Forced moves or good tricks in design space? Landmarks in the evolution of neural mechanisms for action selection. Adaptive Behavior 15 (1) (2007). pp. 9-31.

[21] V.G. Red'ko. Models of cognitive evolution: Initial steps. Proceedings of the 6th International Conference on Neural Network and Artificial Intelligence (ICNNAI'2010). Brest, Belarus, 1-4 June, 2010, pp. 133-139.

[22] M.V. Butz, E. Shirinov, K. Reif. Selforganizing sensorimotor maps plus internal motivations yield animal-like behavior. Adaptive Behavior 18 (3-4) (2010). pp. 315337.

[23] G.A. Beskhlebnova, V.G. Red'ko. Model of formation generalized notions by autonomous agents. Proceedings of 4-th International conference of cognitive science. Tomsk, Russia, 22-26 June 2010, vol. 2, pp. 174-175 (In Russian).

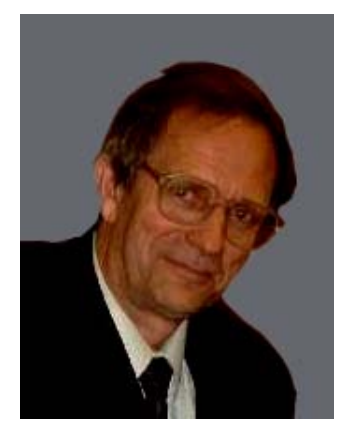

Red'ko Vladimir Georgievich is a Deputy Director for Research of Center of Optical Neural Technologies, Scientific Research Institute for System Analysis, Russian Academy of Sciences. V.G. Red'ko graduated from the Moscow Institute of Physics and Technology in 1971. He is the doctor of sciences (physics and mathematics). He is the author of more than 150 scientific publications, including two monographs.

His scientific interests includes: the problem of origin of human intelligence, cognitive evolution, models of adaptive behavior, neuroinformatics. 